\title{
Competition Between Antiferromagnetic Order and Spin-Liquid Behavior in the Two-Dimensional Periodic Anderson Model at Half-Filling
}

\author{
M. Vekić, ${ }^{1}$ J.W. Cannon, ${ }^{2}$ D.J. Scalapino, ${ }^{3}$ R.T. Scalettar, ${ }^{1}$ and R.L. Sugar ${ }^{3}$ \\ ${ }^{1}$ Department of Physics, University of California, Davis, CA 95616 \\ ${ }^{2}$ Physics Department, Centenary College, 2911 Centenary Blvd., Shreveport, LA 71104 \\ ${ }^{3}$ Department of Physics, University of California, Santa Barbara, CA 93106
}

(August 9, 2018)

\begin{abstract}
We study the two-dimensional periodic Anderson model at half-filling using quantum Monte Carlo (QMC) techniques. The ground state undergoes a magnetic order-disorder transition as a function of the effective exchange coupling between the conduction and localized bands. Low-lying spin and charge excitations are determined using the maximum entropy method to analytically continue the QMC data. At finite temperature we find a competition between the Kondo effect and antiferromagnetic order which develops in the localized band through Ruderman-Kittel-Kasuya-Yosida interactions.
\end{abstract}

PACS numbers: 75.20.Hr, 75.30.Et, 75.30.Mb, 75.40.Mg

Typeset using REVTEX 
The periodic Anderson model (PAM) [1] describes a localized $f$-band of strongly correlated electrons hybridized with a $d$-band of free conduction electrons. As the temperature, filling, and Hamiltonian parameters are varied, there is a competition between the Ruderman-Kittel-Kasuya-Yosida (RKKY) interaction [2] and the Kondo effect [3]. The RKKY interaction favors ordering the magnetic moments of the localized $f$-band, while the Kondo effect screens the localized magnetic moments and quenches the magnetic interaction through the formation of singlets between the two bands 国. The Kondo effect is expected to dominate for large exchange coupling between the conduction electrons and the local moments. For smaller values of the exchange interaction, instead, the local moments order, in most cases antiferromagnetically.

It is believed that this behavior qualitatively describes the competition between magnetic ordering and singlet formation in a number of the heavy fermion materials. Additionally, several small-gap semiconducting compounds involving either rare-earth or actinide metals [5] can be well described by the PAM. These systems exhibit local moment behavior at high temperatures. At low temperatures the hybridization of the local moments with the conduction band leads to magnetic ordering and the formation of a small energy gap or, in some cases, to a Kondo insulator with no long-range magnetic order.

The competition between the RKKY interaction and the Kondo effect has been studied within the framework of the two-impurity Anderson model [6 [10], the one-dimensional PAM [11] and the Kondo lattice [12], and in infinite dimensions [13]. Here we present QMC results for the two-dimensional PAM at half-filling. This is an interesting model since it exhibits various types of insulating states ranging from an antiferromagnetic insulator to a Kondo insulator or, if the $f$ - $d$ hybridization dominates, a simple band-insulator.

The Hamiltonian for the two-dimensional PAM is

$$
\begin{aligned}
\hat{H}= & -t \sum_{\langle i, j\rangle \sigma}\left(d_{i \sigma}^{\dagger} d_{j \sigma}+d_{j \sigma}^{\dagger} d_{i \sigma}\right)+\epsilon_{d} \sum_{i \sigma} n_{i \sigma}^{d}+U_{f} \sum_{i}\left(n_{i \uparrow}^{f}-\frac{1}{2}\right)\left(n_{i \downarrow}^{f}-\frac{1}{2}\right) \\
& +\epsilon_{f} \sum_{i \sigma} n_{i \sigma}^{f}-V \sum_{i \sigma}\left(d_{i \sigma}^{\dagger} f_{i \sigma}+f_{i \sigma}^{\dagger} d_{i \sigma}\right) .
\end{aligned}
$$

Here $t$ is the hopping parameter in the $d$-band, $V$ is the hybridization energy between the 
bands, $U_{f}$ is the Coulomb repulsion in the $f$-band, $\epsilon_{f}$ and $\epsilon_{d}$ are the energy levels of the $d$ and $f$-band respectively, and $n_{i \sigma}^{d} \equiv d_{i \sigma}^{\dagger} d_{i \sigma}$ and $n_{i \sigma}^{f} \equiv f_{i \sigma}^{\dagger} f_{i \sigma}$ are the density operators for the two bands. In the following we will set $t=1$ and consider the symmetric case $\left(\epsilon_{f}=0\right)$ at half-filling $\left(\epsilon_{d}=0\right)$.

The zero temperature behavior of the PAM along the $U_{f}=0$ and $V=0$ axes in the $U_{f^{-}} V$ phase diagram is simple. In the former case, we have a "band-insulator" as the hybridization $V$ opens up a gap where the dispersionless $f$ electron level crosses the conduction band. In the latter case we have a set of individual local moments completely decoupled from the conduction band. When $V$ and $U_{f}$ are both non-zero, a qualitative understanding of the zero temperature order-disorder transition can be obtained from a comparison of the RKKY energy scale $J_{\mathrm{RKKY}} \sim J^{2} / W$ and the Kondo energy scale $T_{K} \sim W e^{-W / J}$, where $J \sim V^{2} / U_{f}$ and $W$ is the bandwidth. For small values of $J$, long-range RKKY driven antiferromagnetic order will set in. As the exchange coupling $J$ is increased beyond a fixed value, we expect that the order will be quickly suppressed due to the formation of spin singlets between the two bands. The above argument suggests a functional form of the phase boundary with $U_{f} \propto V^{2}$.

In order to determine whether the ground state of the PAM does have long-range antiferromagnetic order, we have carried out a finite size scaling analysis of the equal time spin-spin correlations obtained with QMC to calculate the staggered $f$-orbital magnetization $m_{f}$ in the thermodynamic limit. When this quantity is non-zero, the ground state of the PAM has long-range antiferromagnetic order. In addition, we have evaluated non-equal time correlations to extract the dynamic $f$-orbital spin and charge susceptibilities as well as the low temperature dependence of the total uniform spin and charge susceptibilities in order to determine the spin and charge gaps. For an antiferromagnetic Mott insulator the charge gap is finite but there are gapless Goldstone antiferromagnetic spin excitations. However, in a Kondo insulator there are gaps for all excitations (with the charge gap larger than the spin gap) and consequently no long-range order. In the PAM we expect the small exchange interaction phase to be well described by a Mott insulator with antiferromagnetic order, 
while the larger exchange interaction phase should be well described by a Kondo insulator with spin-liquid behavior. In addition, in the limit of a large exchange interaction there is a cross-over to a band-insulator regime where the spin and charge gaps become identical.

At finite temperature we find two different behaviors depending on whether the parameters are such that the ground state is in the order or disordered part of the phase diagram. For small values of the exchange coupling we find a metallic behavior, with a Kondo resonance developing in the $f$-band density of states for temperatures below the Kondo temperature $T_{K}$. This resonance is then suppressed at a lower temperature, leading to insulating behavior, by the formation of a gap when the antiferromagnetic correlations reach the size of the system. The staggered spin susceptibility tends to diverge at the same temperature at which the charge gap forms in $N^{f}(\omega)$. The uniform spin susceptibility displays a maximum below $T_{K}$ and tends to saturate at a finite value as $T$ goes to zero. On the other hand, the uniform charge susceptibility also displays a maximum but then decays quickly to zero, indicating the existence of a charge gap. For large values of the exchange coupling the $f$-band density of states shows an insulating gap for all finite temperatures and the absence of antiferromagnetic order. Both the uniform $q \rightarrow 0$ spin and charge susceptibilities decay to zero as the temperature is lowered, indicating the presence of a gap.

In order to determine the $U_{f^{-}} V$ boundary separating the antiferromagnetically ordered from the disordered phase we have calculated the $f$-band spin correlation function

$$
c^{f}\left(l_{x}, l_{y}\right)=\frac{1}{N} \sum_{i}\left\langle m_{i+l}^{f} m_{i}^{f}\right\rangle,
$$

with $m_{i}^{f}=n_{i \uparrow}^{f}-n_{i \downarrow}^{f}$, and the $f$-band antiferromagnetic structure factor

$$
S^{f}(\pi, \pi)=\sum_{l}(-1)^{l_{x}+l_{y}} c^{f}\left(l_{x}, l_{y}\right),
$$

as functions of temperature and system size $N$. In particular, for a given lattice size, we found that $S^{f}(\pi, \pi)$ saturates for large values of $\beta$. We have used the saturated values along with the spin-wave scaling forms

$$
m_{f}^{2}=\frac{3 S^{f}(\pi, \pi)}{N}+O\left(\frac{1}{\sqrt{N}}\right)
$$


and

$$
m_{f}^{2}=3 c^{f}(\sqrt{N} / 2, \sqrt{N} / 2)+O\left(\frac{1}{\sqrt{N}}\right)
$$

to extract the staggered magnetization $m_{f}^{2}$ of the $f$-band. Figure 1 shows the results of these calculations for two values of the hybridization $V=1.0$ and $V=1.2$ with $U_{f}=4$. The system sizes ranged from $4 \times 4$ to $8 \times 8$ lattices. For $V=1.0$, the intercept of a linear fit to the points gives a finite value for $m_{f}^{2}$ in the infinite system. However, for $V=1.2$, $S^{f}(\pi, \pi)$ does not appear to scale with system size $N$ and, as shown in Fig. 1, we conclude that $m_{f}^{2}=0$. Similar conclusions can be reached by scaling $c^{f}(\sqrt{N} / 2, \sqrt{N} / 2)$ according to the spin-wave theory result. In Fig. 1 we show that the extrapolated value for $m_{f}^{2}$ obtained from Eq.(可) is consistent with the value obtained from $S^{f}(\pi, \pi)$ for $V=1.0$. Again, for $V=1.2$ it appears that $m_{f}^{2}=0$.

We have carried out a similar scaling analysis for several values of $U_{f}$ and in Fig. 2 we show the $U_{f}-V$ phase diagram obtained in this manner. The boundary is shown as a solid line passing through the three points obtained from the QMC data at $U_{f}=2,4$, and 6 . For small values of $J \sim V^{2} / U_{f}$ we have carried out a mean-field calculation assuming that $\left\langle n_{i \sigma}^{f}\right\rangle=\frac{1}{2}+\sigma \frac{m^{f}}{2}(-1)^{i}$. The dotted curve in Fig.2 shows where the mean-field $m_{f}$ vanishes. The antiferromagnetic region obtained from MFT is significantly larger than that found from QMC due to the effect of fluctuations which are neglected in MFT.

In order to extract dynamic properties of the system, we have also calculated imaginarytime properties. Using a maximum entropy procedure [14] to analytically continue the QMC data, we have determined the density of states and the imaginary part of the spin and charge dynamic susceptibilities. We have inverted the integral relation relating the single particle Green's function $G_{i j}^{f}(\tau) \equiv-\left\langle T_{\tau} c_{i}(\tau) c_{j}^{\dagger}(0)\right\rangle$ to the density of states $N^{f}(\omega)$,

$$
G_{i i}^{f}(\tau)=-\int_{-\infty}^{\infty} d \omega \frac{N^{f}(\omega) e^{-\tau \omega}}{1+e^{-\beta \omega}}
$$

In Fig. 3 we show $N^{f}(\omega)$ for several values of $\beta$ for $U_{f}=4$ and $V=0.75$. As the temperature is lowered from $\beta=4$ to $\beta=12$ we see a Kondo resonance peak developing at the Fermi level, 
corresponding to the screening of the local moments by the conduction electrons. However, for finite values of $V$, as the temperature is further lowered, we find that the Kondo peak is suppressed until at $\beta=20$ a well developed gap is formed. On the same lattice size and for the same range of temperatures we find that the antiferromagnetic structure factor grows rapidly before saturating to a finite value at around $\beta=20$. Thus, we associate the gap with the long-range antiferromagnetic order which sets in at the same temperature for this lattice size. For larger values of $V$ the behavior of $N^{f}(\omega)$ is quite different, since we do not find any resonance peak at $\omega=0$ but rather an insulating gap is always present at all temperatures. It is found that this gap grows with $V$ for any fixed $U_{f}$.

We have also analytically continued the imaginary-time $f$-orbital spin and charge correlation functions $\chi_{i i}^{f}(\tau) \equiv\left\langle T_{\tau} m_{i}^{f}(\tau) m_{i}^{f}(0)\right\rangle$ and $\Pi_{i i}^{f}(\tau) \equiv\left\langle T_{\tau} n_{i}^{f}(\tau) n_{i}^{f}(0)\right\rangle$ in order to calculate the imaginary part of the dynamic spin and charge susceptibilities $\operatorname{Im} \chi^{f}(\omega)$ and $\operatorname{Im} \Pi^{f}(\omega)$, respectively. It can be shown that $\operatorname{Im} \chi^{f}(\omega)$ is related to $\chi_{i i}^{f}(\tau)$ by

$$
\chi_{i i}^{f}(\tau)=-\int_{-\infty}^{\infty} d \omega \frac{\operatorname{Im} \chi^{f}(\omega) e^{-\tau \omega}}{1-e^{-\beta \omega}}
$$

with a similar expression relating $\operatorname{Im} \Pi^{f}(\omega)$ to $\Pi_{i i}^{f}(\tau)$. In Fig. 4(a) we show $-\operatorname{Im} \chi^{f}(\omega)$ for several choices of $V$ on a $6 \times 6$ lattice. The spin gap only opens for values of $V$ above the critical point, where there is no antiferromagnetic long-range order. In Fig. 4(b) we show $-\operatorname{Im} \Pi^{f}(\omega)$ with the same parameters as in Fig. 4(a). In this case it is clear that the charge gap $\Delta_{c}$ is present also for values of $V$ below the critical point. In the inset we report the value of the ratio of the spin gap to the charge gap $\Delta_{s} / \Delta_{c}$ as a function of $V$. We find that $\Delta_{c}>\Delta_{s}$ for all values of $V$. However, for the larger values of $V$ we can clearly see that the two gaps tend to be equal. This behavior is an indication that the system crosses into a band-insulator regime.

Evidence for charge and spin gaps can also be seen in the temperature dependence of the uniform charge and spin susceptibilities. The spin susceptibility is defined by

$$
\chi_{\text {tot }}(q=0)=\int_{0}^{\beta} d \tau\langle m(\tau) m(0)\rangle
$$


and the charge susceptibility is similarly defined by

$$
\Pi_{\text {tot }}(q=0)=\int_{0}^{\beta} d \tau\left[\langle n(\tau) n(0)\rangle-\langle n\rangle^{2}\right]
$$

Here $m=\frac{1}{N} \sum_{i}\left(n_{i \uparrow}-n_{i \downarrow}\right)$ and $n=\frac{1}{N} \sum_{i}\left(n_{i \uparrow}+n_{i \downarrow}\right)$, with $n_{i \sigma}=n_{i \sigma}^{f}+n_{i \sigma}^{d}$. In Fig. 5 we show $\chi_{\text {tot }}$ and $\Pi_{\text {tot }}$ versus temperature for several values of the hybridization energy $V$ at a fixed $U_{f}=4$. For smaller values of $V$ we find that $\Pi_{\text {tot }}$ becomes very small at a finite temperature, while $\chi_{\text {tot }}$ peaks at a temperature close to the one at which we find the Kondo resonance in $N^{f}(\omega)$ and then approaches a finite value at $T=0$. This behavior is consistent with the fact that for $V=1.0, \operatorname{Im}^{f}(\omega)$ has a gap but $\operatorname{Im} \chi^{f}(\omega)$ is gapless.

In conclusion, we have shown that for small values of $V^{2} / U t$ the ground state of the PAM is an insulator with long-range antiferromagnetic order characterized by a finite charge gap and gapless spin excitations. As $V^{2} / U t$ increases the long-range order is destroyed and the system exhibits a spin-liquid behavior. The spin-liquid state is characterized by both a spin gap and a charge gap with $\Delta_{c}>\Delta_{s}$. When the hybridization $V$ increases further and $V^{2} / U t$ becomes large, the system crosses over to a band-insulating state in which $\Delta_{s}$ approaches $\Delta_{c}$. Work is currently in progress to determine the behavior of the doped system.

We thank M.P.A. Fisher, R.M. Fye, J. Freericks, M. Jarrell, and A.W. Sandvik for helpful conversations. The numerical calculations were performed primarily on the Cray C-90 at the San Diego Supercomputer Center. This work was supported by the National Science Foundation under grants No. DMR92-06023 and No. PHY89-04035 (Institute for Theoretical Physics) (M.V. and R.T.S.), the Los Alamos National Laboratory under the LACOR grant No. UC-94-2-A-213 (M.V.), and the Department of Energy under grant No. DE-FG03-85ER45197 (D.J.S. and R.L.S.). 


\section{REFERENCES}

[1] P.W. Anderson, Phys. Rev. 124, 41 (1961).

[2] C. Kittel, Quantum Theory of Solids (Wiley, New York, 1963).

[3] J. Kondo, Solid State Physics 23, 183 (1969).

[4] P.A. Lee, T.M. Rice, J.W. Serene, L.J. Sham, and W. Wilkins, Comments Condens. Matter Phys. 12, 99 (1986).

[5] T.E. Mason, G. Aeppli, A.P. Ramirez, K.N. Clausen, C. Broholm, N. Stücheli, E. Bucher, and T.T.M. Palstra, Phys. Rev. Lett. 69, 490 (1992); B. Bucher, Z. Schlesinger, P.C. Canfield, and Z. Fisk, Phys. Rev. Lett. 72, 522 (1994).

[6] R.M. Fye, J.E. Hirsch, and D.J. Scalapino, Phys. Rev. B 35, 4901 (1987).

[7] P. Coleman, Phys. Rev. B 35, 5072 (1987).

[8] B.A. Jones, C.M. Varma, and J.W. Wilkens, Phys. Rev. Lett. 61, 125 (1988); B.A. Jones and C.M. Varma, Phys. Rev. B 40, 324 (1989); B.A. Jones, B.G. Kotliar, and A.J. Millis, Phys. Rev. B 39, 3415 (1989).

[9] O. Sakai, Y. Shimizu, and T. Kasuya, Solid State Commun. 75, 81 (1990).

[10] R.N. Silver, J.E. Gubernatis, and D.S. Sivia, Phys. Rev. Lett. 65, 496 (1990); H.L. Neal, Phys. Rev. Lett. 66, 818 (1991); M. Jarrell, J.E. Gubernatis, and R.N. Silver, Phys. Rev. B 44, 5347 (1991); J.Bonča and J.E. Gubernatis, Phys. Rev. B 47, 13137 (1993).

[11] R.M. Fye, Phys. Rev. B 41, 2490 (1990); R. Blankenbecler, J.R. Fulco, W. Gill, and D.J. Scalapino, Phys. Rev. Lett. 58, 411 (1987); T. Nishino and K. Ueda, Phys. Rev. B 47, 12451 (1993).

[12] R.M. Fye and D.J. Scalapino, Phys. Rev. Lett. 65, 3177 (1990); R.M. Fye and D.J. Scalapino, Phys. Rev. B 44, 7486 (1991). 
[13] M. Jarrell, H. Akhlaghpour, and Th. Pruschke, Phys. Rev. Lett. 70, 1670 (1993); S.J. Sun, M.-F. Yang, and T.-M. Hong, Phys. Rev. B 48, 16127 (1993).

[14] R.N. Silver, D.S. Sivia, and J.E. Gubernatis, Phys. Rev. B 41, 2380 (1990); R.N. Silver, J.E. Gubernatis, D.S. Sivia, and M. Jarrell, Phys. Rev. Lett. 65, 496 (1990); M. Vekić and S.R. White, Phys. Rev. B 47, 1160 (1993). 


\section{FIGURES}

FIG. 1. The square of the staggered magnetization $m_{f}^{2}$ for various $\sqrt{N} \times \sqrt{N}$ lattices as a function of $1 / \sqrt{N}$. The solid symbols are extracted using Eq.(四) and the open symbols using Eq.(5). The circles are for $V=1.0$ and the triangles for $V=1.2$. Here $U_{f}=4$ and $\beta=20$. For comparison, the solid square indicates the value of $m_{f}^{2}$ for the $2 \mathrm{D}$ Hubbard model with $U=4$.

FIG. 2. The $U_{f}-V$ phase diagram of the half-filled PAM at $T=0$, showing the boundary between the antiferromagnetic (AF) and spin-liquid (SL) phases. The solid line is a guide to the eye through the QMC points. The dotted line is the MFT boundary.

FIG. 3. The $f$-band density of states $N^{f}(\omega)$ on a $6 \times 6$ lattice with $U_{f}=4$ and $V=0.75$ for several temperatures. The dashed line is for $\beta=4$, the dashed-dotted line for $\beta=12$ and the solid line for $\beta=20$.

FIG. 4. The imaginary part of (a) the spin susceptibility $-\operatorname{Im} \chi^{f}(\omega)$ and (b) the charge susceptibility $-\operatorname{Im} \Pi^{f}(\omega)$ for $\beta=16$ on a $6 \times 6$ lattice with $U_{f}=4$. The ratio of the spin and charge gaps $\Delta_{s} / \Delta_{c}$ versus $V$ is shown in the inset.

FIG. 5. The uniform (a) spin susceptibility $\chi_{\text {tot }}(q=0)$ and (b) charge susceptibility $\Pi_{\text {tot }}(q=0)$ as a function of temperature $T$ on a $6 \times 6$ lattice with $U_{f}=4$. 


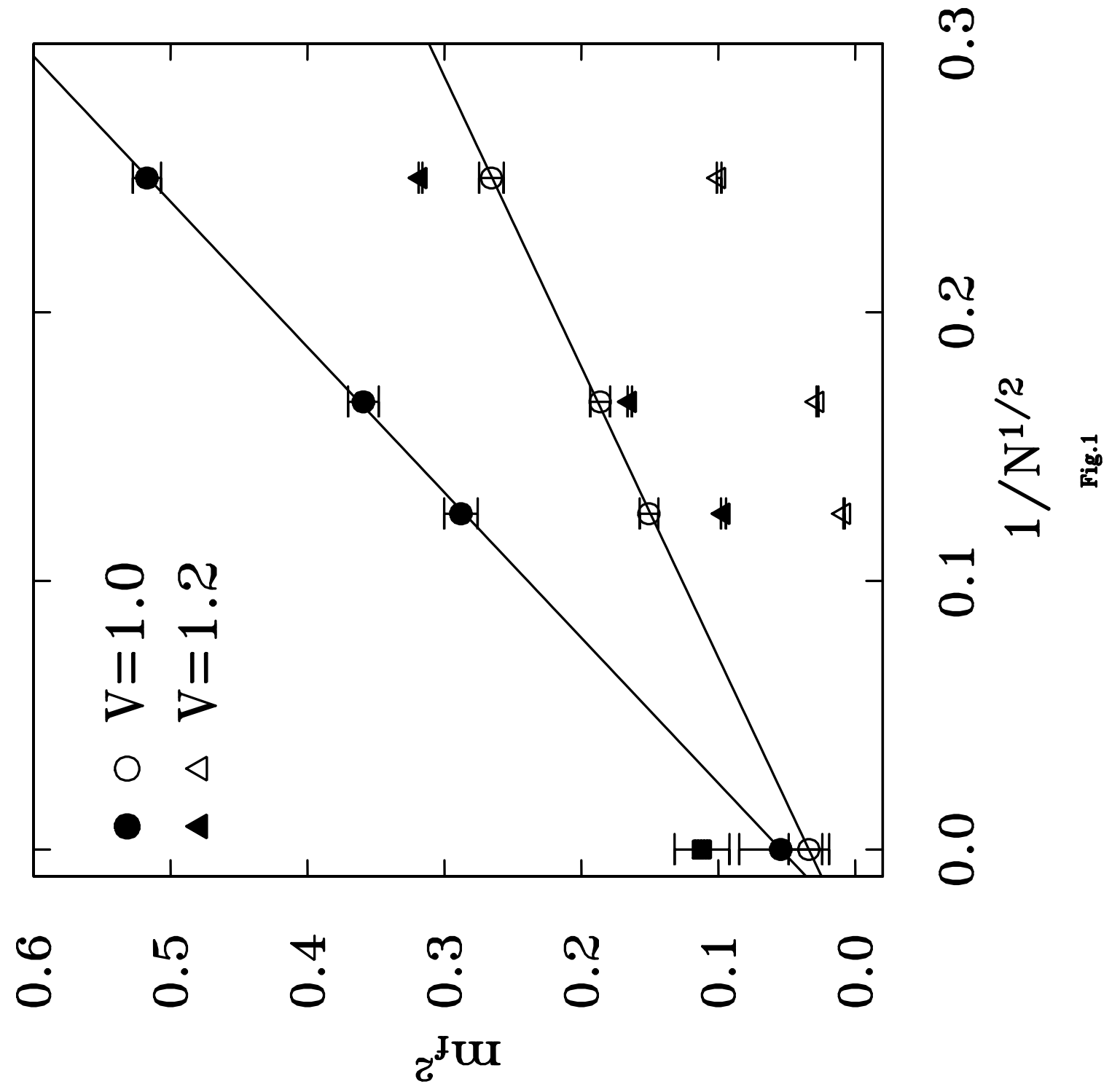




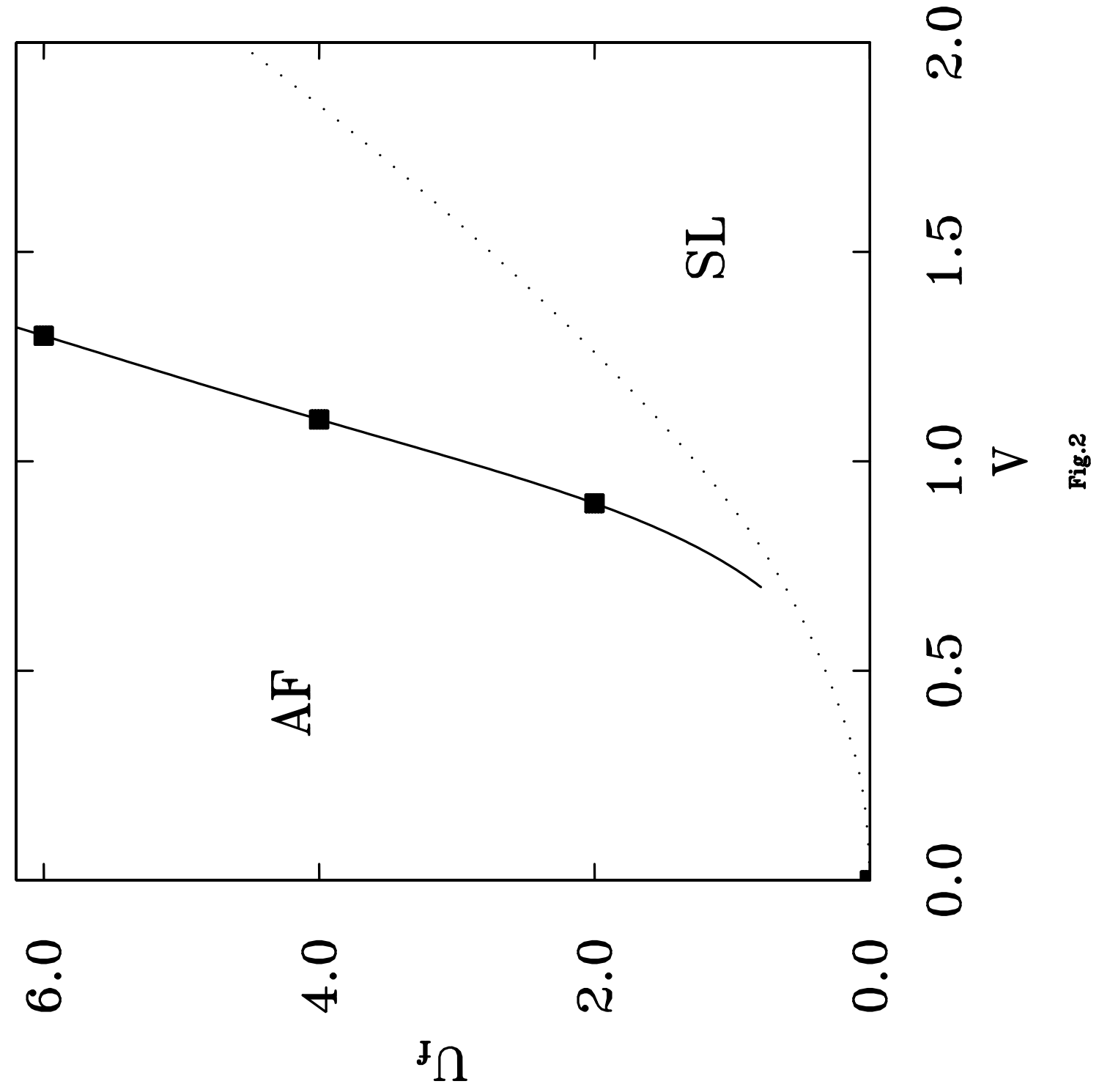




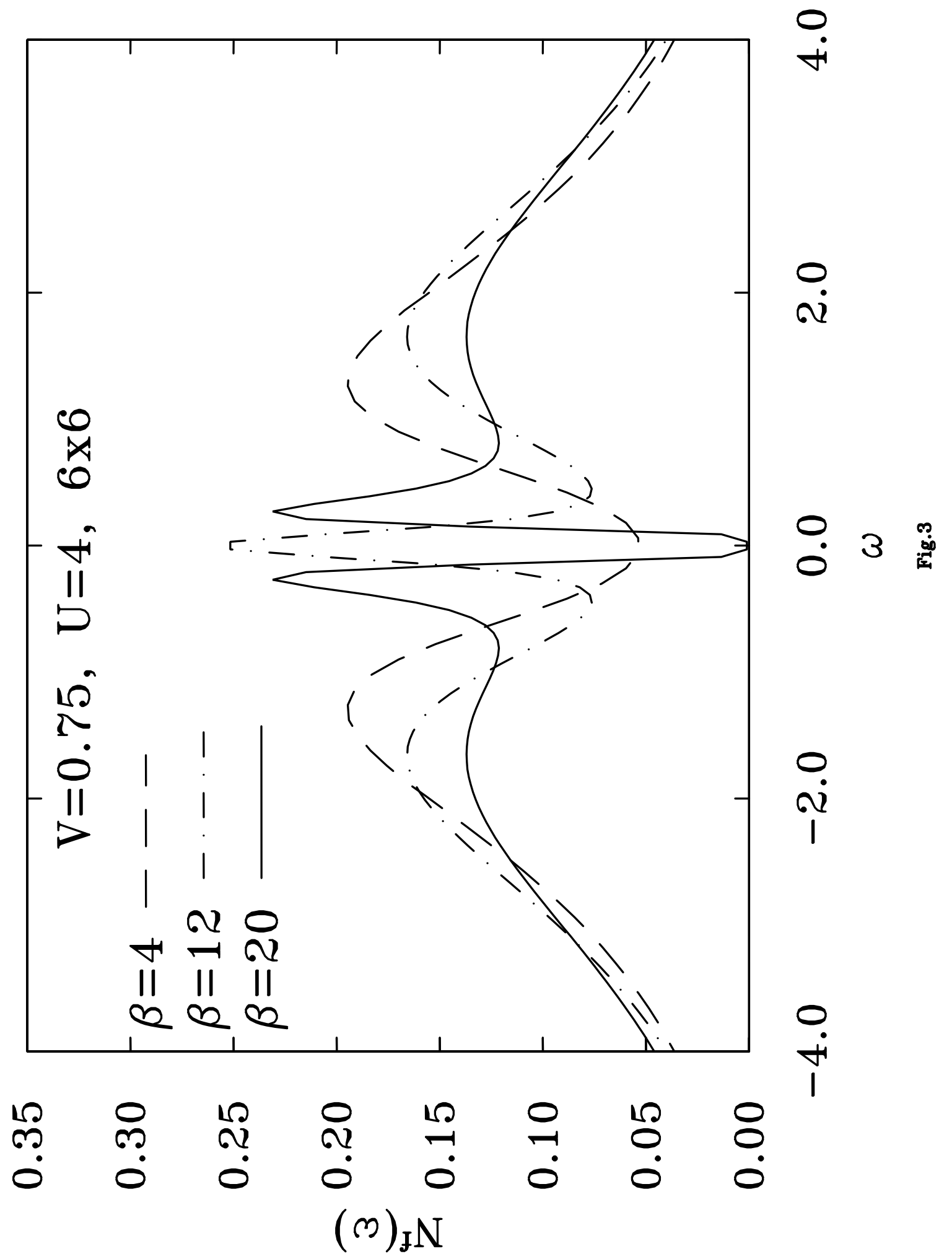




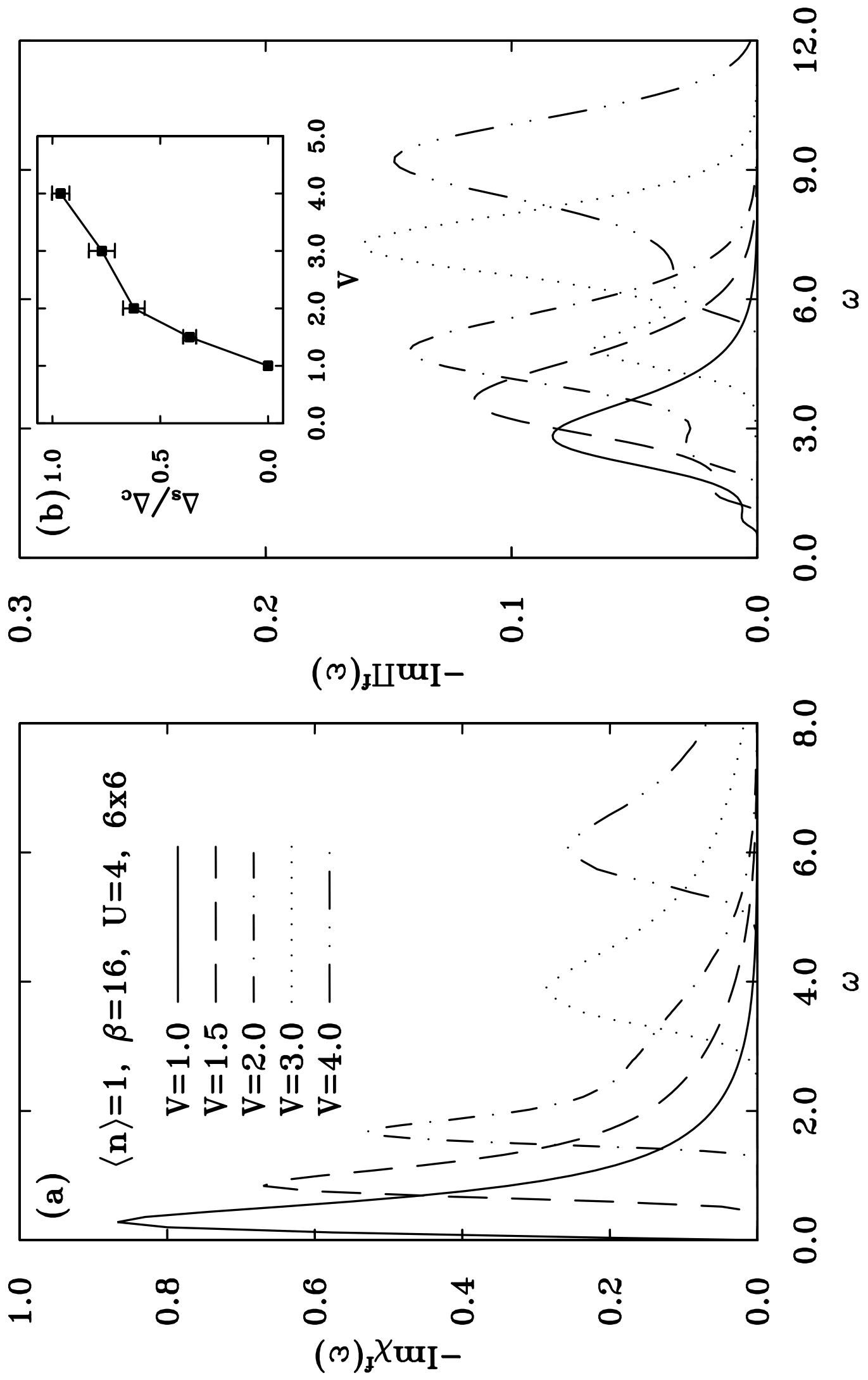




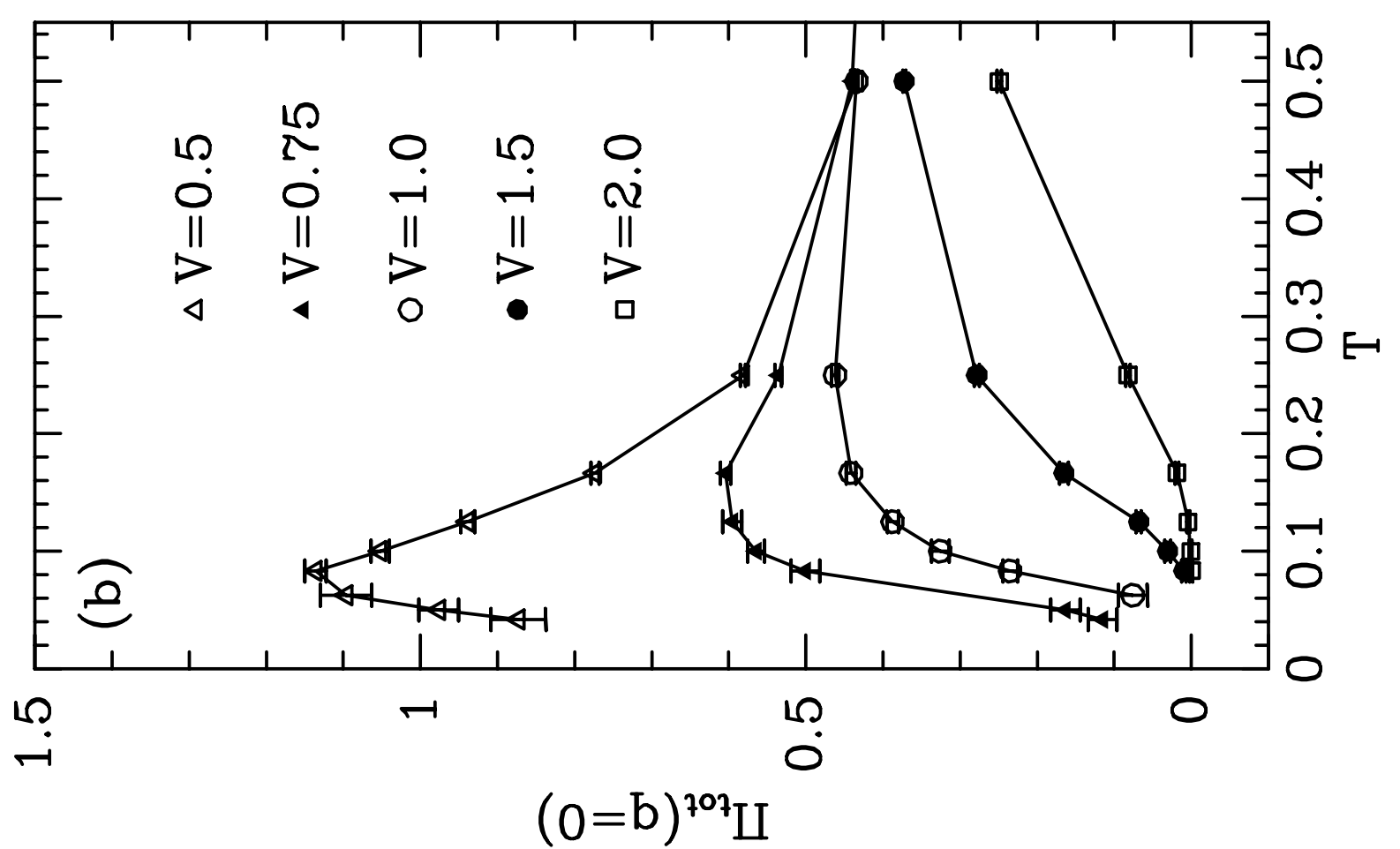

10

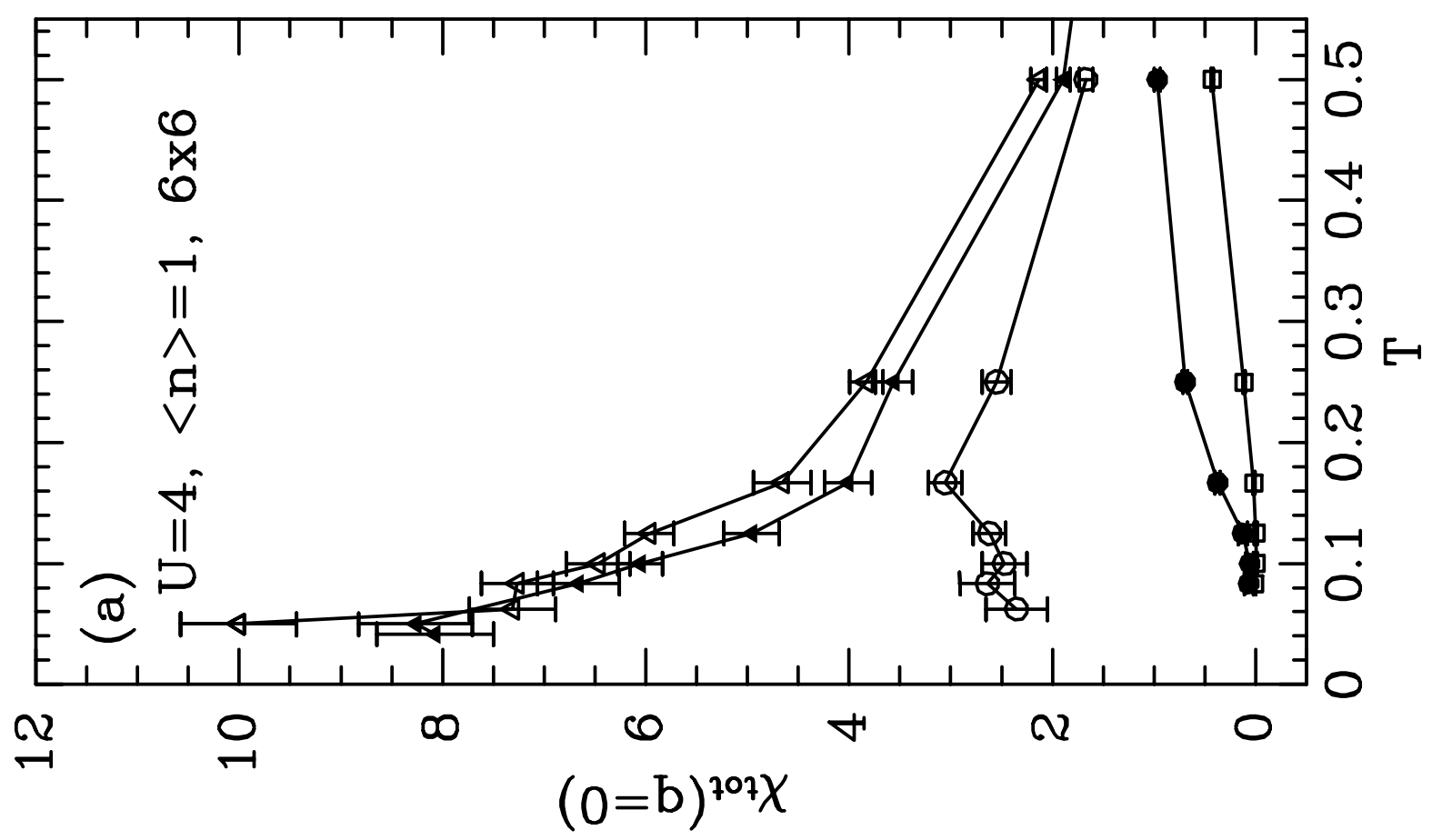

